\title{
Renal transplantation
}

\author{
R S Trompeter
}

End stage renal disease is a rare condition in children. It has a prevalence of $20-50$ children under the age of 15 years per million child population in those European countries with the largest population. The loss of renal function progresses through several stages defined by clinical characteristics and related to the degree of remaining residual function. Chronic renal insufficiency defines the stage in which residual function has not fallen to less than $30 \%$ of normal. Chronic renal failure describes renal function less than $30 \%$ with a residual potential glomerular filtration rate less than $15 \%$ of normal. End stage renal failure defines the point at which dialysis treatment or renal transplantation begins.

The causes of primary renal disease leading to chronic renal failure vary according to country. In general, however, congenital and familial conditions are more common than acquired renal disease. Reflux nephropathy (pyelonephritis) and some form of renal dysplasia or anatomical defect are responsible for more than $50 \%$ of cases, whereas acquired glomerulonephritis accounts for up to $30 \%$.

Data presented in the 17th Combined Report on Regular Dialysis and Transplantation of Children in Europe confirmed the rapid development of continuous ambulatory peritoneal dialysis used in $23 \%$ of patients who commenced treatment for end stage renal disease in 1986, compared with $10 \%$ in $1981 .^{1}$ As the first method of treatment of end stage renal failure, however, renal transplantation increased from 2 to only $5 \%$ over the same period. Nevertheless it is encouraging to read that almost $50 \%$ of all children on the registry file aged less than 15 years at the end of 1986 had a functioning renal allograft. Taken from the same report actuarial survival for patients engrafted between 1983 and 1986 showed an $87 \%$ survival at three years for live related donor kidneys and $65 \%$ for cadaveric grafts. These figures reflected the wide ranging results obtained in the many centres surveyed, as a consequence of variations in clinical practice especially in the use of immunosuppressive drugs. Recent analysis from centres in the United Kingdom using comparable clinical management and immunosuppressive regimens would suggest at least an $80 \%$ graft survival of first cadaver donor kidneys at one year and virtually $100 \%$ success for live related donor grafts. $^{2} 3$

Renal transplantation has therefore become firmly established as the preferred treatment for children suffering from end stage renal failure.
There are many major differences between paediatric and adult renal transplantation. Foremost is the primary cause of renal failure, with a high incidence of children in whom there is urological abnormality. Before renal transplantation, the recipient native kidneys are removed only in exceptional circumstances. Intractable nephrotic syndrome, severe arterial hypertension, and infection associated with vesicoureteric reflux that cannot be managed by medical treatment are such examples.

Recognition that an adult donor kidney can be safely engrafted intra-abdominally in very small infants has necessitated the use of large more proximal recipient blood vessels such as the aorta and inferior vena cava. ${ }^{4}$ Although adult cadaver kidneys are more readily available, the use of paediatric organs is preferable. The high incidence of complications with kidneys from infants less than 2 years of age, however, especially arterial and venous thrombosis, argues against their use.

Increasing experience in the perioperative management and meticulous attention to fluid and electrolyte balance has reduced the problem of intratransplant sequestration and drainage of a significant proportion of the blood volume and cardiac output of a small recipient, after vascular anastomosis. This has significantly reduced graft loss secondary to arterial or venous thrombosis. $^{5}$

The greater emphasis in some centres on living related donor allografts, has resulted in substantial improvement in outcome with renal transplantation in recipients under the age of 30 months. ${ }^{6}$ Results are acceptable and therefore may not justify a prolonged wait for a small cadaveric kidney.

The controversial question, Should kidney transplants ever be performed in the first year of life in those children born with renal failure? is frequently asked. The answer is never clear, is always controversial, and confounded by ethical issues concerned with infant bonding and psychomotor development during the first year of life. There are undoubtedly some infants for whom extending life by dialysis and transplantation is not in the best interests of the patient or their family. However, in the face of technical advance the decision not to treat is always a difficult one. Current trends in some paediatric renal units in the United Kingdom would suggest that up to $25 \%$ of the population with end stage renal failure is under 5 years of age. This together with a prevailing move towards transplantation before a dialysis requirement has been realised places enormous demands on 
the carers. Only a constant and improving supply of cadaver donor kidneys can guarantee that advances will be made in this area.

\section{Patient preparation before transplant}

Review of the results of cadaveric renal allograft survival would suggest that there is little universal agreement as to the selection of the best available human leucocyte antigen (HLA) match, that is, tissue typing. ${ }^{7}$ Indeed the efficacy of HLA matching is controversial. At any moment in time the demand for cadaveric organs exceeds supply and it is therefore rarely feasible for many transplant units to pay strict attention to HLA matching. Immunological damage to the renal allograft is mediated by both humoral and cellular mechanisms. Acute humoral rejection results in a vasculitic catastrophe occurring when kidneys are transplanted into hosts in whom preformed cytotoxic antibodies exist, targeted against the blood groups $\mathrm{ABO}$ or HLA associated antigens present in the transplanted organ. ${ }^{8}$ Chronic humoral rejection is also principally targeted against graft endothelium and invariably leads to inexorable graft loss. Rejection pathways are initiated by activation of a population of recipient antidonor $T$ lymphocyte clones that recognise donor histocompatibility antigens in the graft. Humoral rejection is refractory to current antirejection treatment and may indeed be ultimately best treated by prevention through accurate-that is, complete-tissue matching.

Therefore on the day of transplantation a cross match test is mandatory. $\mathrm{ABO}$ typing and cross matches between recent and historic recipient serum and donor $\mathrm{T}$ and $\mathrm{B}$ lymphocytes are obtained in order to exclude the presence of preformed recipient antidonor, class I HLA cytotoxic antibodies capable of producing acute humoral rejection. Class I antigens coded for by the A, B, and C loci, are glycoproteins detected on all cells of the transplantable organs, and peripheral blood lymphocytes are the best readily available source of cells for testing. The distribution of class II or DR antigens is confined primarily to endothelium and the dendritic cells in most lymphoid tissue. The total time for HLA typing is between four and six hours, and conceptually matching as a means of improving graft survival is attractive as incompatibility for these antigens may well be the major stimulus for the generation of the immune response against a renal allograft. ${ }^{9}$

\section{Immunosuppression}

No consensus has developed on how to achieve optimal immunosuppression, and indeed many individual centres employing different protocols report excellent results for both graft and patient survival. A biological approach to immunosuppression is mandatory, using several agents simultaneously each of which is directed at a different mechanism of activation of allograft rejection. Triple or quadruple immunosuppression using corticosteroids, azathioprine, cyclosporin, and a polyclonal antibody have become standard practice. Their benefit allows for the use of a low dosage of each individual agent, thus obtaining maximum immunosuppressive benefit with minimal toxicity. Detailed discussion of individual dosage regimens is beyond the scope of this review but a brief description of each immunosuppressive agent is given.

Prednisolone used from the time of transplantation interferes with $T$ cell activation by blocking the signals mediated by the lymphokine interleukin I, and remains the potent drug in preventing early acute rejection. Cyclosporin, now in clinical use for almost 10 years, blocks the elaboration and release of interleukin II thus depriving the activated $\mathrm{T}$ cell of the stimulus necessary to sustain activation. The antimetabolite azathioprine is the nitroimidazole derivative of 6-mercaptopurine and is used to inhibit both DNA and RNA synthesis. This drug will therefore inhibit cell proliferation of those lymphocytes not already affected by prednisolone and cyclosporin. Low dosage prednisolone, cyclosporin, and azathioprine are probably ineffective in blocking the activity of already activated $T$ cells and thus these drugs are relatively incapable of suppressing an already established acute rejection. Polyclonal antibody preparations such as antithymocyte globulin are therefore used as antirejection prophylaxis in many centres. Monoclonal preparations directed against specific components of the human $\mathrm{T}$ lymphocyte complex are still experimental and experience to date is restricted to their use in adult patients. ${ }^{10}$

The principal target of cyclosporin is the kidney itself and nephrotoxicity can occur at any time in the post transplant period. The early reversible form of toxicity leads to decreased blood flow because of vasoconstriction; failure to reduce the dosage promptly may result in more permanent damage characterised by interstitial fibrosis and glomerulosclerosis. ${ }^{11}$ Cyclosporin is strongly lipophilic and thus measured therapeutic concentrations correlate poorly with toxicity in some patients. Renal injury secondary to other nephrotoxic drugs or acute renal failure may potentiate the toxic effects of cyclosporin. Extrarenal complications include tremor, gingival hypertrophy, and hirsutism, the latter particularly troublesome in children.

\section{Allograft rejection}

The clinical signs and symptoms of acute rejection are protean but are likely to include fever, swelling and tenderness of the graft, hypertension, oliguria, and a measurable deterioration in renal function. The histopathology of cellular rejection is characterised by a dense infiltration of $T$ cells within the medullary regions of the graft. Allograft rejection remains the single most common cause of graft loss for any group of renal transplant recipients. Fortunately most acute cellular rejection episodes respond to bolus corticosteroid treatment, more resistant rejection may respond to antithymocyte globulin. This is a polyclonal antibody preparation derived from either a 
horse or rabbit immunised with human lymphocytes. The antibodies are not specific, although directed primarily against lymphocytes. Its value in treating steroid resistant rejection is well established even though adverse reactions including anaphylaxis, thrombocytopenia, neutropenia, and phlebitis are common.

\section{Future developments}

The disease from which a recipient of an allografted kidney develops renal failure may affect the outcome in two ways. Firstly, the disease may be such that the recipient's life is threatened, alternatively the grafting may be put in jeopardy by the recurrence of the original disease in the grafted kidney. ${ }^{12}$ For Wilms' tumour transplantation within one year of native nephrectomy was associated with a recurrence of disseminated disease. Patient survival with bilateral tumours may be as low as $38 \%$, death being associated with infection, perhaps aggravated by chemotherapy and irradiation. ${ }^{13}$ The recurrence of certain types of glomerulonephritis remains a problem. Lesions of focal segmental glomerulosclerosis and a nephrotic syndrome may recur in almost a quarter of all cases especially in those children in whom there was a rapid onset of renal failure. Improved techniques for the early diagnosis of infection in the immunocompromised patient and a better understanding of the transmission of glomerulonephritis are clearly needed to improve both patient and graft survival in this group. Uncorrected congenital abnormalities of bladder storage and abnormal bladder emptying (for example, posterior urethral valves) also appear to adversely affect graft survival. ${ }^{14}{ }^{15} \mathrm{An}$ earlier understanding of bladder physiology could improve the results in these patients.

Anti-HLA antibodies may occur as a result of a failed transplant or blood transfusions and persist in high titres for many years. Approximately 20 to $30 \%$ of patients in the United Kingdom, of whom few are children, currently awaiting renal transplantation have these antibodies. These patients are classified as highly sensitised and are thus difficult to transplant. Preventing sensitisation by the transplantation of a well matched live related or cadaver donor kidney without HLA antigens against which the recipient has made antibodies would be ideal but is not feasible given the poor supply of cadaveric organs. ${ }^{16}$ Strategies for the removal of anti-HLA antibodies using plasma exchange and immunosuppression are potentially useful but remain experimental and have a risk of antibody resynthesis. ${ }^{17}$

The ideal immunosuppressive strategy for tissue transplantation is one that permits the development of long term immunologic tolerance with minimal interference with normal body processes. With more careful prescription the side effects of prednisolone and cyclosporin have become less of a clinical problem, although they remain significant. The future must rest on the production of a natural human antilymphocyte monoclonal antibody.

The management of children with end stage renal disease is frequently complicated by growth retardation and renal osteodystrophy. The concept that growth potential at the time of transplantation, as indicated by bone age, is an important factor for growth after transplant, and that recipients with a bone age greater than 12 years grow minimally despite successful transplantation has been challenged ${ }^{18}$ as postpubertal recipients had significant height increment after transplantation. Impaired linear growth therefore reflects several factors including growth potential achieved at the time of transplant, poor allograft function, and corticosteroid dosage required for immunosuppression. Poor linear growth before transplantation undoubtedly has a great influence on adult height. However, the loss of growth potential during pubertal development may be even more important. ${ }^{19}$ Evaluation of the development of hormonal changes during puberty is needed in order to further understand the characteristics of growth.

In addition to concerns about growth retardation and pubertal delay, children who receive transplants must also cope with the tasks of growing emotionally and psychologically. Evidence of low esteem, defective social adaptation, and non-compliance with immunosuppression is present in many children assessed by child psychiatrists and supported by skilled social workers. ${ }^{20}$ There is no doubt that the quality of life of a transplant recipient is far superior to that of a dialysis patient, but that quality is not ideal. Medicines must be taken daily in order to prevent rejection, hospital attendances are necessary for physical examination and blood tests, and admission to hospital to treat complications of transplant surgery and the side effects of immunosuppression are not uncommon. Ultimately all renal transplant recipients must live with the uncertainty that their graft might fail at any time and this has been compared with the 'Damocles syndrome' often encountered in the treatment of patients with malignant disease. ${ }^{21}$ The high rate of psychiatric morbidity in children with end stage renal failure and their parents is intimately linked to the fact that most families have coping difficulties, and suggests the need for skilled intervention.

Renal transplantation is technically feasible and is successful for most children. Of approximately 3500 potential renal transplant recipients currently listed on the United Kingdom Transplant Register, 80 are children under 16 years. The challenges that remain to all involved in the care of the children with end stage renal failure are not only to provide a therapeutic environment that will ensure good quality of life but also to guarantee that the present level of service can be maintained.

1 Ehrich JHH, Rizzoni G, Broyer M, et al. Combined report on regular dialysis and transplantation of children in Europe 1987. London: Springer-Verlag, 1988:33-41.

2 Rigden S. Cyclosporin therapy in children. Cyclosporin. Issues of today. Oxford: Medical Education Services, 1986: of today.

3 Fitzpatrick $M$, Fernando ON, Barratt TM, Dillon MJ, Trompeter RS. Triple immunosuppression and intravenous or oral cyclosporin A, azathioprine and prednisovenous or oral cyclosporin A, azathioprine and predniso-
lone in children receiving renal transplants. Proceedings of the European Society for Paediatric Nephrology. Manchester the European
1988:49. 
4 Miller LC, Lum CT, Bock GH, Simmons RL, Najarian JS, Mauer SM. Transplantation of the adult kidney into the very small child. Am f Surg 1983;145:243-7.

5 Trompeter RS, Bewick M, Haycock GB, Chantler C. Renal transplantation in young children. Lancet 1983;i:313-5.

6 So SKS, Chang P, Najarian JS, Mauer MS, Simmons RL Nevins TE. Growth and development in infants after transplantation. F Pediatr 1987;110:343-50.

7 Opelz G. Importance of HLA antigen splits for kidney transplant matching. Lancet $1988 ;$ ii:61-4.

8 Morris PJ. The immunology of rejection. In: Morris PJ, ed. Kidney transplantation. Principles and practice. 2nd ed. London: Grune and Stratton, 1984:15-32.

9 Ting A. HLA and renal transplantation. In: Morris PJ, ed. Kidney transplantation. Principles and practice. 2nd ed. London: Grune and. Stratton, 1984:159-80.

10 Norman DI. An overview of the use of monoclonal antibody OKT3 in renal transplantation. Transplant Proc 1988; 20:1248-52.

11 Strom TB, Loertscher R. Cyclosporine-induced nephrotoxicity: inevitable and intractable? $N$ Eng $\mathcal{f}$ Med 1984;311:728-9.

12 Cameron JS. The effect of the recipients disease on the results of transplantation. Kidney Int 1983;23(suppl 14):s24-33.

13 Penn I. Renal transplantation for Wilms' tumour: report of 20 cases. F Urol 1979;122:793-4.
14 Churchill BM, Sheldon CA, McLorie G, Arbus GA. Factors influencing patient and graft survival in 300 cadaveric pediatric renal transplants. F Urol 1988;140:1129-33.

15 Reinberg Y, Gonzalez R, Fryd D, Mauer SM, Najarian JS. The outcome of renal transplantation in children with posterior urethral valves. F Urol 1988;140:1491-3.

16 Broyer M, Gagnadoux MF, Buerton D, Pascal B, Busson M, Hors J. Importance of HLA - A, B matching in kidney
transplantation in children. Transplantation 1980;30:310.

17 Palmer A, Welsh $K$, Gjorstrup $P$, Taube $D$, Bewick $M$, Thick M. Removal of anti-HLA antibodies by extracorporeal immunoadsorption to enable renal transplantacorporeal immunoadsorption. Lancet 1989;i:10-2.

18 Bosque M, Munian A, Haycock GB, Chantler C. Growth after renal transplantation. Arch Dis Child 1983;58:110-4.

19 Van Diemen-Steenvoorde R, Donckerwolcke RA, Brackel H, Wolff ED, Jong MCJW. Growth and sexual maturation in children after kidney transplantation. 7 Pediatr 1987; 110:351-6.

20 Korsch BM, Negrete VF. Psychosocial adaptation of children with ESRD: factors affecting rehabilitation. In: Fine RN, with ESRD: factors affecting rehabilitation. In: Fine $\mathrm{RN}$, Gruskin AB, eds. End stage renal disease
delphia: WB Saunders, 1984;553-9.

21 Koocher GP, O'Malley JE. The Damocles syndrome. New York: McGraw Hill, 1981 . 